\title{
ARTICLE
}

\section{Leptin and incident type 2 diabetes: risk or protection?}

\author{
M. I. Schmidt • B. B. Duncan • A. Vigo • J. S. Pankow • \\ D. Couper $\cdot$ C. M. Ballantyne $\cdot$ R. C. Hoogeveen • \\ G. Heiss $\cdot$ For the ARIC Investigators
}

Received: 7 February 2006 / Accepted: 20 May 2006 / Published online: 19 July 2006

(C) Springer-Verlag 2006

\begin{abstract}
Aims/hypothesis The aim of this study was to investigate the association of leptin levels with incident diabetes in middle-aged adults, taking into account factors purportedly related to leptin resistance.

Subjects and methods We conducted a case-cohort study (570 incident diabetes cases and 530 non-cases) representing the 9-year experience of 10,275 participants of the Athero-
\end{abstract}

M. I. Schmidt • B. B. Duncan • A. Vigo

Graduate Studies Program in Epidemiology, School of Medicine,

Federal University of Rio Grande do Sul,

Porto Alegre, RS, Brazil

M. I. Schmidt $•$ B. B. Duncan $\cdot$ G. Heiss

Department of Epidemiology, School of Public Health,

University of North Carolina,

Chapel Hill, NC, USA

\section{J. S. Pankow}

Division of Epidemiology, School of Public Health,

University of Minnesota,

Minneapolis, MN, USA

D. Couper

Department of Biostatistics, School of Public Health, University of North Carolina,

Chapel Hill, NC, USA

C. M. Ballantyne $\cdot$ R. C. Hoogeveen

Department of Medicine, Baylor College of Medicine,

Houston, TX, USA

M. I. Schmidt $(\square)$

School of Medicine, UFRGS,

R. Ramiro Barcelos, 2600/414,

Porto Alegre, RS 90035-003, Brazil

e-mail: mischmidt@orion.ufrgs.br sclerosis Risk in Communities Study. Plasma leptin was measured by direct sandwich ELISA.

Results In proportional hazards models adjusting for age, study centre, ethnicity and sex, high leptin levels (defined by sex-specific cut-off points) predicted an increased risk of diabetes, with a hazard ratio (HR) comparing the upper with the lower quartile of 3.9 (95\% CI 2.6-5.6). However, after further adjusting additionally for obesity indices, fasting insulin, inflammation score, hypertension, triglycerides and adiponectin, high leptin predicted a lower diabetes risk (HR $=0.40,95 \%$ CI $0.23-0.67)$. Additional inclusion of fasting glucose attenuated this protective association (HR $=0.59,95 \%$ CI $0.32-1.08, p<0.03$ for linear trend across quartiles). In similar models, protective associations were generally seen across subgroups of sex, race, nutritional status and smoking, though not among those with lower inflammation scores or impaired fasting glucose (interaction $p=0.03$ for both).

Conclusions/interpretation High leptin levels, probably reflecting leptin resistance, predict an increased risk of diabetes. Adjusting for factors purportedly related to leptin resistance unveils a protective association, independent of adiponectin and consistent with some of leptin's described protective effects against diabetes.

Keywords Inflammatory markers $\cdot$ Leptin .

Leptin resistance $\cdot$ Risk factors $\cdot$ Type 2 diabetes

$\begin{array}{ll}\text { Abbreviations } \\ \text { ARIC } & \text { Atherosclerosis Risk in Communities } \\ \text { CRP } & \text { C-reactive protein } \\ \text { HR } & \text { hazard ratio } \\ \text { OR } & \text { odds ratio }\end{array}$




\section{Introduction}

Diabetes has become a major health priority worldwide. The epidemic rise in obesity and type 2 diabetes has stimulated new avenues of investigation, such as the role of inflammation in the progression from obesity to diabetes. Along these lines, we found that a mild chronic state of inflammation precedes and predicts incident type 2 diabetes $[1,2]$. These associations have been confirmed in different ethnic groups, and analyses have been expanded to include various circulating molecules with inflammatory and metabolic properties, as recently reviewed [3].

One important adipocyte-derived signalling molecule operating within this context, leptin [4-7], has been little investigated in epidemiological studies of incident type 2 diabetes. In Japanese Americans, greater baseline leptin levels were associated with an increased risk of developing diabetes in men but not in women [8]. In Mauritius, risk of diabetes increased with increasing levels of leptin, with a tendency to plateau at high levels [9]. More recently, elevated leptin levels have been found to predict worsening of glucose intolerance in middle-aged white adults [10].

These risk associations could result from direct noxious effects of leptin on glucose metabolism, though such effects have not been consistently found across different experimental models [6, 11, 12]. In fact, many animal studies suggest a protective role for leptin in the development of diabetes [13-17]. Thus, as leptin levels are elevated in obese individuals, suggesting resistance to its action in the control of food intake and energy expenditure [18], it is possible that the strong associations observed between leptin and diabetes in the above epidemiological studies result from resistance to central and/or peripheral leptin actions in obese subjects progressing to diabetes.

Leptin's protective actions may not have been noted in epidemiological studies of type 2 diabetes because high leptin levels are usually accompanied by leptin resistance. One way of overcoming this problem is to adjust leptindiabetes associations not only for obesity, which reflects directly the pool of leptin production, but also for central fat distribution, hyperinsulinemia/insulin resistance and subclinical inflammation, all known to be linked to a metabolic dysregulation purportedly related to leptin resistance [19-21].

Thus, the purpose of this study was to examine the relationship of leptin levels with incident diabetes, both when minimally adjusted and when taking into account the effects of obesity, WHR, fasting insulin, inflammation markers, and other components of the metabolic syndrome purportedly related to leptin resistance. Analyses were based on a large case-cohort study designed to investigate chronic inflammation and incident diabetes in white and
African-American adults participating in the Atherosclerosis Risk in Communities (ARIC) study.

\section{Subjects and methods}

Subjects

Between 1987 and 1989 the ARIC study recruited a population-based cohort of 15,792 men and women aged 45-64 years from four US communities [22]. All subjects were invited to return to three clinic visits, at approximately 3-year intervals, at which incident diabetes was ascertained. Human subjects' research review committees at the involved institutions approved the study, and all participants gave written consent.

We chose the same case-cohort design previously used to investigate the role of inflammation in the development of diabetes [2], which permits evaluation of the association between leptin and incident diabetes within the context of a sample well characterised with respect to inflammation markers. As an OGTT was not performed at baseline, we defined both prevalent and incident diabetes on the basis of the following: a reported physician diagnosis, use of glucose-lowering medications, a fasting ( $\geq 8 \mathrm{~h}$ ) glucose of $\geq 7.0 \mathrm{mmol} / 1$, or a non-fasting glucose of $\geq 11.1 \mathrm{mmol} / \mathrm{l}$. The date of diabetes incidence was estimated by linear interpolation using glucose values at the ascertaining visit and the previous one, as previously described [2]. Prior to sampling, we excluded 2,018 participants with prevalent diabetes, 95 members of minority ethnic groups with small numbers, 853 individuals who did not return to any follow-up visit, 26 with no valid diabetes determination at follow-ups, seven with restrictions on stored plasma use, 12 with missing baseline anthropometric measurements, and 2,506 participants in previous ARIC case-control and case-cohort studies involving cardiovascular disease for whom stored plasma was either previously exhausted or held in reserve (Fig. 1). This resulted in a final sample of 10,275 individuals $(75 \%$ of those in the full cohort without diabetes at baseline), of whom 1,155 (11.2\%) were ascertained as developing diabetes during follow-up. From these 10,275 eligible members of this baseline cohort, we selected and measured several analytes on ethnicity-stratified (50\% white, 50\% African-American) random samples of both cases of incident diabetes and of eligible members of the full cohort, a total of 1,198 individuals. A few of the incident cases of diabetes overlapped with the cohort random sample, and a few were selected only via the cohort sample. Of those sampled, we excluded 45 for incomplete fasting $(<8 \mathrm{~h})$ or for not having values for all covariates, and 27 identified as non-cases but having 2 -h glycaemia $\geq 11.1 \mathrm{mmol} / 1$ during the OGTT performed at the last follow-up visit, leaving a total of 
Fig. 1 Selection of case-cohort participants

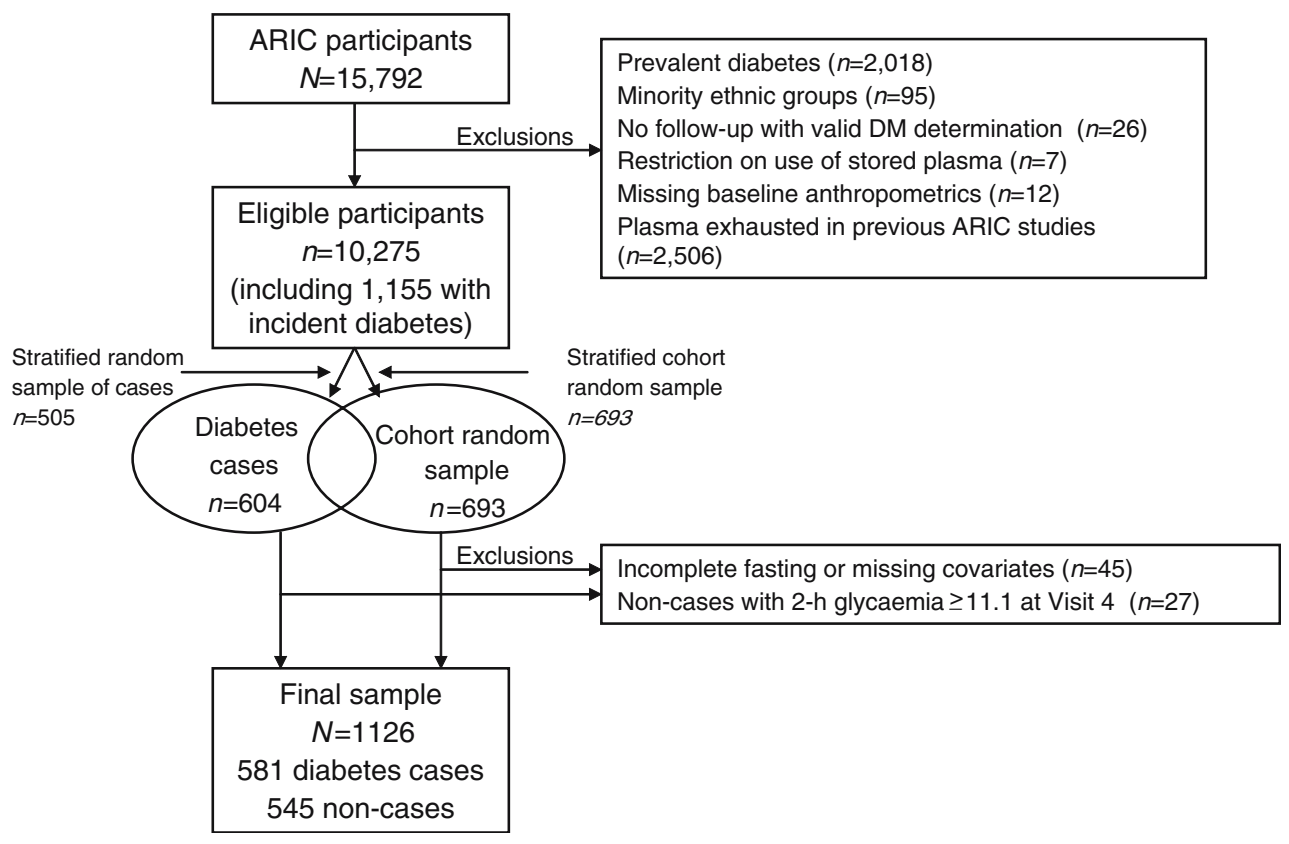

1,126 subjects, including 581 diabetes cases and 545 noncases, for analysis. The cohort random sample contained $4.2 \%$ of eligible white participants and $13.6 \%$ of eligible African-Americans. Among those with incident diabetes, $40 \%$ of eligible white and $70 \%$ of eligible AfricanAmerican cohort participants were included. For the purpose of maintaining the same number of participants across models of the association between leptin and incident diabetes, we excluded an additional 26 subjects from the proportional hazards analyses owing to missing information on covariates.

\section{Laboratory measurements}

We measured glucose at baseline and at follow-up visits by a hexokinase method, fasting serum insulin by non-specific RIA. We measured waist girth at the umbilical level and hip circumference at the maximum hip girth in order to obtain the WHR. We defined parental history of diabetes as a report of diabetes in either parent, and BMI as weight divided by height squared. The definitions and methods for other baseline measurements (height, weight, smoking status, systolic blood pressure, hypertension, physical activity, triglycerides, HDL cholesterol, insulin, white blood cell count, fibrinogen, IL-6, sialic acid, C-reactive protein [CRP], orosomucoid and adiponectin) have been previously reported $[1,2]$.

Leptin was measured in duplicate by direct sandwich ELISA (Linco Research, St Charles, MI, USA) at a central laboratory on plasma specimens frozen at baseline. These samples, stored for approximately 15 years at $-70^{\circ} \mathrm{C}$, were thawed and maintained at $4^{\circ} \mathrm{C}$ until measured, no longer than $24 \mathrm{~h}$ later. A reliability coefficient of 0.94 for leptin, expressing between-person: total variance, was obtained analysing replicate pairs of samples drawn at baseline from a subset of 35 subjects.

As in a previous report [2], we created a score, ranging from $0-6$, to indicate low-grade systemic inflammation, attributing 1 point for a value greater than the median of the cohort sample for each of the six inflammation markers: IL-6, CRP, orosomucoid, sialic acid, white cell count and fibrinogen.

\section{Statistical analysis}

Statistical analysis was based on our case-cohort sampling design, weights being defined as the inverse of the ethnicityspecific sampling fractions, thus permitting statistical estimation and inference relevant to the entire cohort. In the cohort random sample, we performed weighted analysis of covariance to compute adjusted means and proportions of socio-demographic variables and risk factors, and weighted Spearman's correlations to describe unadjusted associations between study variables.

The adjusted relative risk of developing diabetes at different quantiles of leptin was estimated in proportional hazards models fitted using SUDAAN (Research Triangle Institute, Research Triangle Park, NC, USA) to account for the case-cohort design, with stratified sampling both from the whole cohort and from the incident diabetes cases [23]. The proportional hazards assumption was examined through plots of martingale and Schoenfeld residuals [24]. To adjust for obesity, WHR, fasting insulin, inflammation markers and other related metabolic variables, we included continuous variables with their quadratic terms, retaining the quadratic terms in the models when statistically significant at the level 
of 0.20. Collinearity across independent variables was investigated with linear regression models, suspicion being raised if a particular variable presented a variance inflation factor $\geq 5$. To test heterogeneity across categories of covariates in the associations between leptin and incident diabetes, we characterised leptin tertiles and covariate categories using indicator variables and employed a chunk test of interaction terms. Analyses were performed using SAS (SAS Institute, Cary, NC, USA) and SUDAAN. A $p$ value of less than 0.05 was considered statistically significant.

\section{Results}

Among incident diabetes cases there were 153 (26\%) white men, 151 (26\%) white women, 76 (13\%) African-American men and 201 (35\%) African-American women. Among non-cases, the corresponding numbers were 117 (21\%), 189 (35\%), 75 (14\%), and 164 (30\%), respectively. Additional characteristics of cases and non-cases have previously been reported $[2,25]$.

The distribution of leptin values was considerably rightskewed, and normality was not achieved despite various transformations. As shown in Table 1, median and interquartile range values varied according to subgroups, particularly across sex and BMI categories.

Also shown in Table 1 are means $(95 \% \mathrm{CI})$ adjusted for age, sex, race and study centre. Similar to the non-parametric comparisons, leptin means were lower in the non-obese, male, white, current smoking and low inflammation subgroups. These differences were generally attenuated after further adjustment for BMI, WHR, fasting insulin, the inflammation score and other metabolic syndrome elements, those for race and smoking groups no longer being statistically significant. Even with this full adjustment, women continued to have four-fold higher leptin levels than men.

Spearman correlations in the cohort random sample for leptin levels with inflammatory and metabolic factors are shown in Table 2. Although leptin correlations were generally of modest magnitude, they were markedly confounded by sex, as can be seen examining these correlations separately in men and women. For example, the correlation between WHR and leptin was -0.05 overall, but 0.40 and 0.44 in men and women, respectively. Furthermore, among women, correlations were frequently stronger, especially those with inflammatory markers. For example, the correlation with CRP was 0.48 in women vs 0.20 in men; that with fibrinogen, 0.35 in women and 0.04 in men.

The median (interquartile range) follow-up was 3.0 (1.75.9) years for those who developed diabetes and 8.9 (8.7-9.0) years for those who did not. Among the incident cases, 499 $(86 \%)$ were ascertained by a fasting glucose of $\geq 7.0 \mathrm{mmol} / \mathrm{l}$, and five $(1 \%)$ by a non-fasting glucose of $\geq 11.1 \mathrm{mmol} / \mathrm{l}$. Independently of their glucose values, 125 (22\%) were additionally ascertained by a reported physician diagnosis or hypoglycaemic medication use. Those who developed diabetes had higher levels of leptin after adjustment for age, sex, race and study site (24.1 vs $16.8 \mathrm{ng} / \mathrm{ml} ; p<0.001)$. After

Table 1 Leptin levels at baseline in the cohort random sample of 693 individuals

\begin{tabular}{|c|c|c|c|c|c|c|c|c|c|}
\hline \multirow[t]{2}{*}{ Variable } & \multirow[t]{2}{*}{ Strata } & \multirow{2}{*}{$\begin{array}{l}\text { Median } \\
(\mathrm{ng} / \mathrm{ml})\end{array}$} & \multirow{2}{*}{$\begin{array}{l}\text { Interquartile } \\
\text { range }\end{array}$} & \multicolumn{3}{|c|}{ Minimal adjustment } & \multicolumn{3}{|c|}{ Full adjustment } \\
\hline & & & & $\begin{array}{l}\text { Mean } \\
(\mathrm{ng} / \mathrm{ml})\end{array}$ & $95 \% \mathrm{CI}$ & $p$ value & $\begin{array}{l}\text { Mean } \\
(\mathrm{ng} / \mathrm{ml})\end{array}$ & $95 \% \mathrm{CI}$ & $p$ value \\
\hline \multicolumn{2}{|l|}{ Overall } & 10.6 & $4.9-21.7$ & 16.9 & $15.4-18.4$ & & 17.1 & $16.0-18.2$ & \\
\hline \multirow[t]{3}{*}{ BMI } & Normal & 8.4 & $3.6-13.5$ & 8.0 & $6.8-9.2$ & & 11.0 & $9.7-12.2$ & \\
\hline & Overweight & 8.2 & $4.5-18.2$ & 15.9 & $14.4-17.4$ & & 15.5 & $14.1-16.9$ & \\
\hline & Obese & 35.2 & $17.0-51.7$ & 36.4 & $31.5-41.3$ & $<0.001$ & 32.1 & $27.4-36.8$ & $<0.001$ \\
\hline \multirow{2}{*}{ Sex } & Female & 17.0 & $10.4-32.9$ & 23.4 & $21.1-25.7$ & & 23.6 & $21.8-25.3$ & \\
\hline & Male & 4.4 & $2.4-7.0$ & 5.9 & $4.9-6.8$ & $<0.001$ & 6.0 & $4.5-7.5$ & $<0.001$ \\
\hline \multirow[t]{2}{*}{ Race } & Black & 20.3 & $6.9-37.1$ & 24.4 & $21.8-27.0$ & & 18.6 & $16.3-20.8$ & \\
\hline & White & 9.8 & $4.7-17.4$ & 15.0 & $13.2-16.8$ & $<0.001$ & 16.7 & $15.2-18.2$ & 0.26 \\
\hline \multirow[t]{3}{*}{ Smoking } & Current & 8.3 & $3.9-16.2$ & 13.3 & $11.1-15.6$ & & 15.2 & $13.2-17.2$ & \\
\hline & Ex & 7.4 & $4.4-17.7$ & 19.1 & $15.6-22.6$ & & 17.7 & $15.1-20.3$ & \\
\hline & Never & 13.8 & $7.4-29.1$ & 17.3 & $15.2-19.3$ & 0.003 & 17.6 & $16.2-19.0$ & 0.14 \\
\hline \multirow[t]{2}{*}{ Inflammation score } & Low & 9.2 & $4.5-16.6$ & 13.0 & $11.8-14.3$ & & 15.9 & $14.8-17.0$ & \\
\hline & High & 16.0 & $6.2-36.8$ & 23.9 & $20.6-27.2$ & $<0.001$ & 19.1 & $17.1-21.2$ & 0.005 \\
\hline
\end{tabular}

The ARIC study, 1987-1999

Results are presented as medians (interquartile range) or adjusted means $(95 \% \mathrm{CI})$

Minimal adjustment: age, sex, centre, race

Full adjustment: age, sex, centre, race, $\mathrm{BMI} \mathrm{BMI}^{2}$, WHR, insulin, insulin ${ }^{2}$, inflammation score, hypertension, triglycerides, triglycerides ${ }^{2}$, glucose

Note that the variable defining the strata in question was not included as a covariable in analyses estimating its strata-specific leptin parameters 
Table 2 Spearman correlations for leptin levels with inflammation and metabolic variables at baseline in the cohort random sample of 693 individuals

\begin{tabular}{|c|c|c|c|c|c|c|}
\hline \multirow[t]{2}{*}{ Variable } & \multicolumn{2}{|c|}{ Overall } & \multicolumn{2}{|c|}{ Women } & \multicolumn{2}{|l|}{ Men } \\
\hline & $r$ & $p$ value & $r$ & $p$ value & $r$ & $p$ value \\
\hline CRP & 0.36 & $<0.001$ & 0.48 & $<0.001$ & 0.20 & 0.003 \\
\hline Orosomucoid & 0.27 & $<0.001$ & 0.43 & $<0.001$ & 0.14 & 0.04 \\
\hline Fibrinogen & 0.26 & $<0.001$ & 0.35 & $<0.001$ & 0.04 & 0.56 \\
\hline IL-6 & 0.19 & $<0.001$ & 0.31 & $<0.001$ & 0.12 & 0.07 \\
\hline Total sialic acid & 0.15 & $<0.001$ & 0.25 & $<0.001$ & 0.04 & 0.52 \\
\hline Fasting insulin & 0.46 & $<0.001$ & 0.65 & $<0.001$ & 0.52 & $<0.001$ \\
\hline BMI & 0.49 & $<0.001$ & 0.75 & $<0.001$ & 0.67 & $<0.001$ \\
\hline Waist circumference & 0.34 & $<0.001$ & 0.71 & $<0.001$ & 0.65 & $<0.001$ \\
\hline WHR & -0.05 & 0.22 & 0.44 & $<0.001$ & 0.40 & $<0.001$ \\
\hline Systolic blood pressure & 0.10 & 0.02 & 0.33 & $<0.001$ & 0.14 & 0.03 \\
\hline Fasting glucose & 0.09 & 0.02 & 0.27 & $<0.001$ & 0.20 & 0.003 \\
\hline Triglycerides & 0.06 & 0.11 & 0.26 & $<0.001$ & 0.19 & 0.005 \\
\hline HDL cholesterol & 0.13 & $<0.001$ & -0.23 & $<0.001$ & -0.15 & 0.03 \\
\hline
\end{tabular}

The ARIC study, 1987-1999

additional adjustment for obesity indices and other elements of the metabolic syndrome, identical to that performed for analyses shown in Table 1, the direction of the association changed, with those who developed diabetes presenting lower leptin levels (14.1 vs $18.3 \mathrm{ng} / \mathrm{ml} ; p<0.001)$.

Given the marked sex differences observed for leptin levels and for leptin correlations with metabolic syndrome variables, we examined associations of leptin with incident diabetes using sex-specific cut-off values for leptin quantiles. Table 3 summarises the hazard ratios and their $95 \%$ CIs for these associations in models with increasing numbers of covariates. The first leptin quartile always serves as the reference category.
A strong, monotonic, positive association was noted between leptin levels and incident diabetes in the model adjusted only for age, sex, race and study centre, the hazard ratio for diabetes being 3.9 (95\% CI 2.6-5.6) comparing extreme leptin quartiles. Addition of BMI to the model (Model 2) abolished this association, suggesting that confounding by obesity explains the apparent risk seen for leptin in the initial model. Further addition of WHR, fasting insulin and the inflammation score (Models 3-5) successively decreased the magnitude of the hazard ratios, higher leptin levels being associated with decreased risk (for the model including these three variables, comparing extreme quartiles, hazard ratio $[\mathrm{HR}]=0.39$, CI $0.23-0.65$ ). Addition

Table 3 HRs and 95\% CIs of incident diabetes for each sex-specific quartile of leptin

\begin{tabular}{|c|c|c|c|c|c|c|c|}
\hline \multirow[t]{2}{*}{ Model } & \multicolumn{2}{|c|}{ 2nd Quartile } & \multicolumn{2}{|c|}{ 3rd Quartile } & \multicolumn{2}{|c|}{ 4th Quartile } & \multirow{2}{*}{$\begin{array}{l}\text { Linear trend } \\
p \text { value }\end{array}$} \\
\hline & HR & $95 \% \mathrm{CI}$ & HR & $95 \% \mathrm{CI}$ & HR & $95 \% \mathrm{CI}$ & \\
\hline Model 1 & 1.60 & $1.05-2.43$ & 2.25 & $1.52-3.35$ & 3.86 & $2.63-5.65$ & $<0.001$ \\
\hline Model 2 & 1.09 & $0.69-1.72$ & 1.10 & $0.70-1.73$ & 1.01 & $0.60-1.69$ & 0.92 \\
\hline Model 3 & 1.07 & $0.68-1.70$ & 0.93 & $0.59-1.48$ & 0.79 & $0.47-1.33$ & 0.23 \\
\hline Model 4 & 0.94 & $0.59-1.52$ & 0.62 & $0.38-1.02$ & 0.41 & $0.23-0.71$ & $<0.001$ \\
\hline Model 5 & 0.94 & $0.59-1.48$ & 0.59 & $0.36-0.94$ & 0.39 & $0.23-0.65$ & $<0.001$ \\
\hline Model 6 & 0.87 & $0.55-1.38$ & 0.57 & $0.35-0.92$ & 0.37 & $0.22-0.63$ & $<0.001$ \\
\hline Model 7 & 0.89 & $0.56-1.41$ & 0.59 & $0.37-0.96$ & 0.40 & $0.23-0.67$ & $<0.001$ \\
\hline Model 8 & 1.06 & $0.63-1.77$ & 0.71 & $0.40-1.25$ & 0.59 & $0.32-1.08$ & 0.03 \\
\hline
\end{tabular}

Case-cohort sample of 1,100 individuals, the ARIC study

Model 1: Adjusted for age, sex and race/centre indicators

Model 2: Model 1 plus BMI, BMI ${ }^{2}$

Model 3: Model 2 plus WHR

Model 4: Model 3 plus ln-insulin and ln-insulin ${ }^{2}$

Model 5: Model 4 plus inflammation score

Model 6: Model 5 plus hypertension and ln-triglycerides, ln-triglycerides ${ }^{2}$

Model 7: Model 6 plus adiponectin

Model 8: Model 7 plus glucose 
of two elements of the metabolic syndrome-hypertension and triglycerides (Model 6) - produced minimal change. The inclusion of adiponectin (Model 7), a cytokine with antidiabetic properties, slightly increased hazard ratios (comparing extreme quartiles, HR=0.40, CI 0.23-0.67). Adding fasting glucose (Model 8) further increased hazard ratios (comparing extreme quartiles, $\mathrm{HR}=0.59$; CI $0.32-1.08$; test for linear trend across quartiles $p=0.03$ ).

Repeat analyses, in which all continuous covariables were modelled as quartiles, revealed a similar pattern of associations for all models (data not shown).

To examine the consistency of these associations across subgroups, leptin was categorised in tertiles, so as to provide an adequate number of incident cases across leptin tertiles in each subgroup. Table 4 shows that men and women presented the same pattern of associations between leptin and incident diabetes as seen overall, although leptin presented somewhat greater risk in minimally adjusted models for men and somewhat greater protection in more adjusted models for women. Although the differences were not statistically significant (interaction $p=0.41$ in the final model), the protective association seen in the final model appeared stronger in women (third vs first tertile leptin, $\mathrm{HR}=0.34,95 \%$ CI $0.18-0.65)$ than in men $(\mathrm{HR}=0.67,95 \%$ CI $0.30-1.53$ ). Figures 2 and 3 show that the pattern of the associations described for the whole sample was generally seen across various other subgroups.

In the minimally adjusted models, a notable exception was that higher levels of leptin were protective in obese participants (interaction $p=0.01$ ). To investigate whether the greater risk associated with higher leptin levels among the non-obese individuals could be explained by residual confounding by BMI, we additionally adjusted for BMI within this stratum. With this adjustment, increased risk was no longer seen for the non-obese (third vs first tertile leptin, HR $=0.94 ; 95 \%$ CI $0.56-1.57$ ), suggesting that the heterogeneity seen across BMI strata in minimal models was largely due to residual confounding by BMI.

In fully adjusted models, additional exceptions to the general pattern were that a protective association was present only in those with higher inflammation scores (interaction $p=0.03$ ) and was notably smaller in those with impaired fasting glucose (interaction $p=0.04$ ).

Further analyses considering cases ascertained in each of the three follow-up visits separately showed similar protective associations, supporting the proportional hazards assumption. Additionally, multiple plots of martingale and Schoenfeld residuals were consistent with this assumption. Collinearity among covariates was examined in all models and judged not to be present to an important degree, as indicated by the fact that the variance inflation factors were never greater than 5 (data not shown).

\section{Discussion}

Our results, which indicate that high levels of leptin are associated with an increased risk of developing diabetes in minimally adjusted models, are consistent with previous epidemiological findings [8-10]. However, that further

Table 4 HRs and 95\% CIs of incident diabetes for each sex-specific tertile of leptin, by sex

\begin{tabular}{|c|c|c|c|c|c|c|c|c|c|c|}
\hline \multirow[t]{3}{*}{ Model } & \multicolumn{5}{|c|}{ Women } & \multicolumn{5}{|l|}{ Men } \\
\hline & \multicolumn{2}{|c|}{ 2nd Tertile } & \multicolumn{2}{|c|}{ 3rd Tertile } & \multirow{2}{*}{$\begin{array}{l}\text { Linear trend } \\
p \text { value }\end{array}$} & \multicolumn{2}{|c|}{ 2nd Tertile } & \multicolumn{2}{|c|}{ 3rd Tertile } & \multirow{2}{*}{$\begin{array}{l}\text { Linear trend } \\
p \text { value }\end{array}$} \\
\hline & HR & $95 \%$ CI & $\mathrm{HR}$ & $95 \%$ CI & & $\mathrm{HR}$ & $95 \% \mathrm{CI}$ & $\mathrm{HR}$ & $95 \% \mathrm{CI}$ & \\
\hline Model 1 & 1.33 & $0.86-2.07$ & 2.53 & $1.65-3.87$ & $<0.001$ & 1.59 & $0.93-2.73$ & 3.07 & $1.84-5.12$ & $<0.001$ \\
\hline Model 2 & 0.77 & $0.47-1.25$ & 0.60 & $0.32-1.12$ & 0.12 & 0.93 & $0.50-1.73$ & 0.91 & $0.45-1.86$ & 0.83 \\
\hline Model 3 & 0.72 & $0.45-1.18$ & 0.50 & $0.28-0.93$ & 0.03 & 0.85 & $0.45-1.60$ & 0.74 & $0.35-1.54$ & 0.43 \\
\hline Model 4 & 0.54 & $0.34-0.88$ & 0.28 & $0.15-0.50$ & $<0.001$ & 0.84 & $0.43-1.64$ & 0.50 & $0.23-1.10$ & 0.06 \\
\hline Model 5 & 0.53 & $0.33-0.85$ & 0.26 & $0.15-0.45$ & $<0.001$ & 0.86 & $0.44-1.69$ & 0.46 & $0.20-1.03$ & 0.04 \\
\hline Model 6 & 0.50 & $0.32-0.80$ & 0.25 & $0.15-0.44$ & $<0.001$ & 0.79 & $0.41-1.53$ & 0.44 & $0.20-0.95$ & 0.02 \\
\hline Model 7 & 0.51 & $0.32-0.82$ & 0.27 & $0.15-0.48$ & $<0.001$ & 0.75 & $0.39-1.44$ & 0.47 & $0.22-1.01$ & 0.04 \\
\hline Model 8 & 0.61 & $0.36-1.05$ & 0.34 & $0.18-0.65$ & $<0.001$ & 0.82 & $0.43-1.59$ & 0.67 & $0.30-1.53$ & 0.35 \\
\hline
\end{tabular}

Case-cohort sample of 1,100 individuals, the ARIC study

Model 1: Adjusted for age, sex and race/centre indicators

Model 2: Model 1 plus BMI, $\mathrm{BMI}^{2}$

Model 3: Model 2 plus WHR

Model 4: Model 3 plus $\ln$-insulin and ln-insulin ${ }^{2}$

Model 5: Model 4 plus inflammation score

Model 6: Model 5 plus hypertension and ln-triglycerides, ln-triglycerides ${ }^{2}$

Model 7: Model 6 plus adiponectin

Model 8: Model 7 plus glucose 
Fig. 2 Heterogeneity in the association of leptin with incident diabetes by sex (a, b; open columns: women, filled columns: men), race (c, d; open columns: African-Americans, filled columns: whites) and smoking (e, f; open columns: smokers, filled columns: nonsmokers). Left panel, minimally adjusted model (adjusted for age, centre, ethnicity and sex); right panel, fully adjusted model (adjusted additionally for BMI, $\mathrm{BMI}^{2}$, WHR, inflammation score, hypertension, triglycerides, triglycerides ${ }^{2}$, fasting glucose, insulin, insulin ${ }^{2}$ ) a

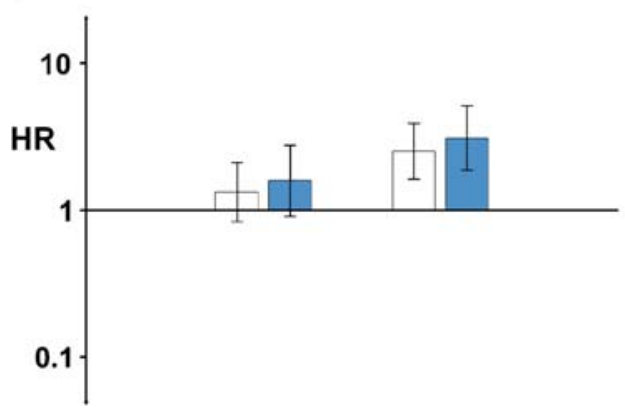

C

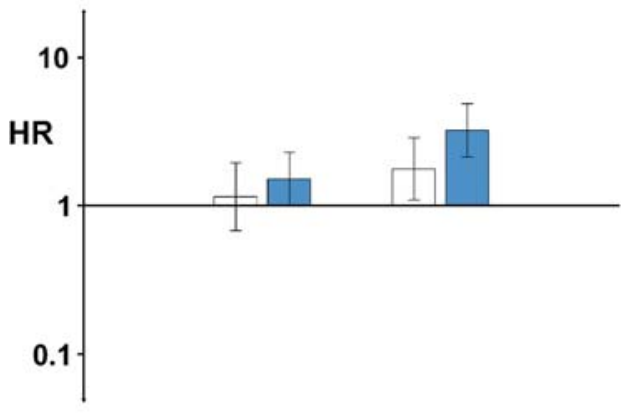

e

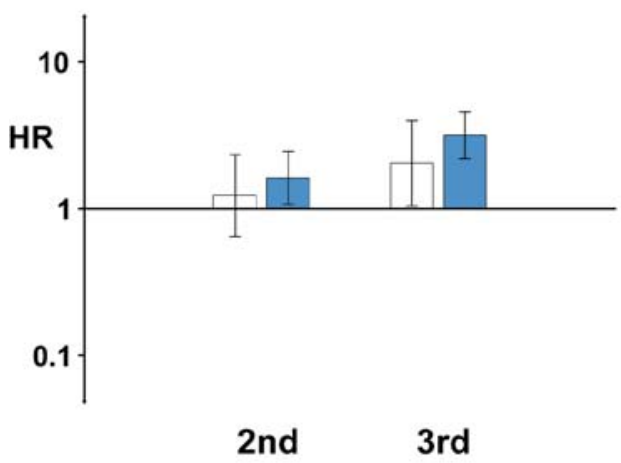

Minimal adjustment b

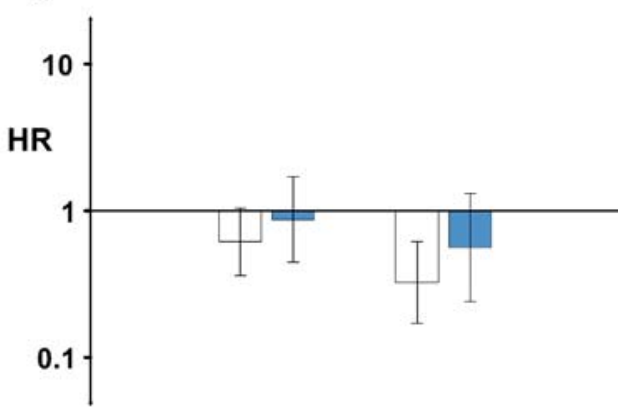

d
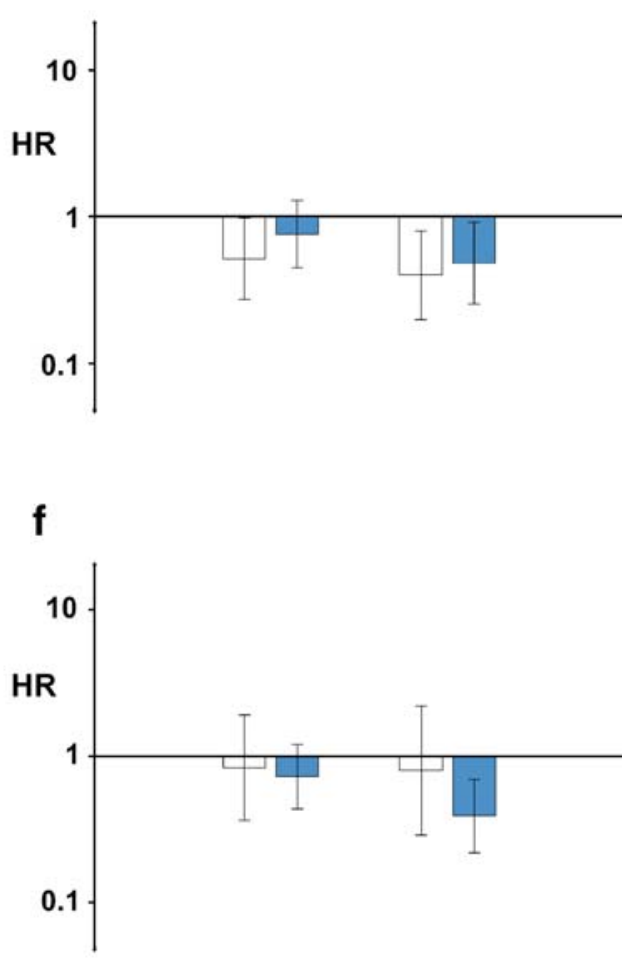

2nd 3rd

Full adjustment

\section{Leptin tertiles}

statistical adjustment for BMI, WHR, fasting insulin, inflammation score, hypertension and triglycerides revealed protective associations - those with highest adjusted leptin levels having only $40 \%$ the incidence of diabetes of those with lowest levels, independently of adiponectin - is novel, and, if confirmed, potentially relevant.

Interestingly, in the Japanese American study (with a relatively small number of incident cases, $n=40$ ), while men with higher leptin levels had an increased risk of diabetes (odds ratio $[\mathrm{OR}]=1.8,95 \% \mathrm{CI} 1.02-3.17$ for a 1-SD change in leptin) in analyses adjusting for total fat, the point estimate found for women, though not statistically signif- icant, was similar in magnitude to that found in the ARIC women $(\mathrm{OR}=0.66,95 \%$ CI $0.30-1.48)$ [8]. In the Mauritian study, after adjustment for obesity indices and metabolic syndrome elements, a complex association was found, with increasing risk at the lower end of the leptin spectrum and a plateau of risk at the higher end of the spectrum [9]. Although differences in study populations may in part explain the heterogeneous findings, for example, ARIC participants were more frequently obese and of AfricanAmerican ancestry, other factors may have been involved. First, our analysis of men and women together used sexspecific cut-off points for leptin, given the four-fold sex 
Fig. 3 Heterogeneity in the association of leptin with incident diabetes by adiposity (a, b; open columns: $\mathrm{BMI} \geq 30 \mathrm{~kg} / \mathrm{m}^{2}$, filled columns $\mathrm{BMI}<30 \mathrm{~kg} / \mathrm{m}^{2}$ ), inflammation (c, d; open columns: low score, filled columns: high score) and baseline impaired fasting glucose (e, f; open columns: absent, filled columns: present). Left panel, minimally adjusted model (adjusted for age, centre, ethnicity and sex); Right panel, fully adjusted model (adjusted additionally for BMI, $\mathrm{BMI}^{2}$, WHR, inflammation score, hypertension, triglycerides, triglycerides ${ }^{2}$, fasting glucose, insulin, insulin ${ }^{2}$ ) a

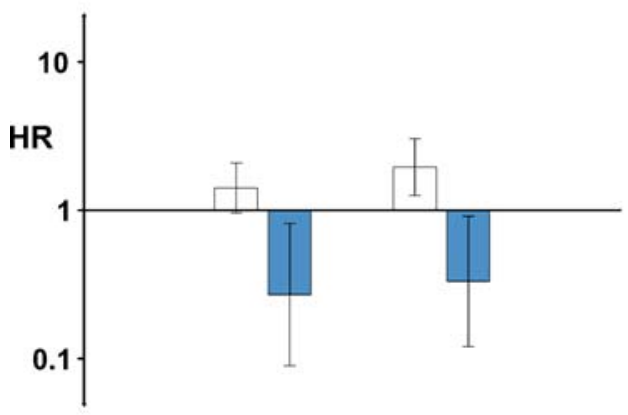

C

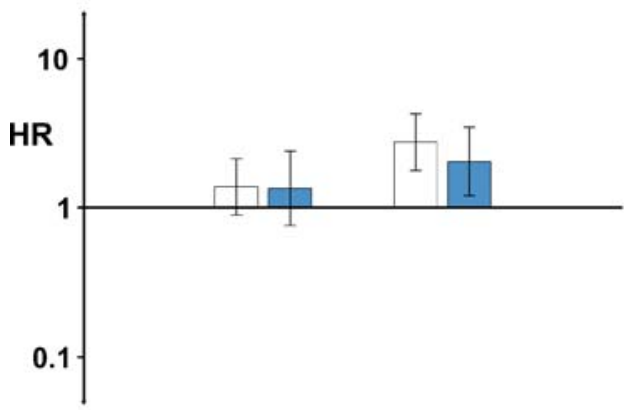

e

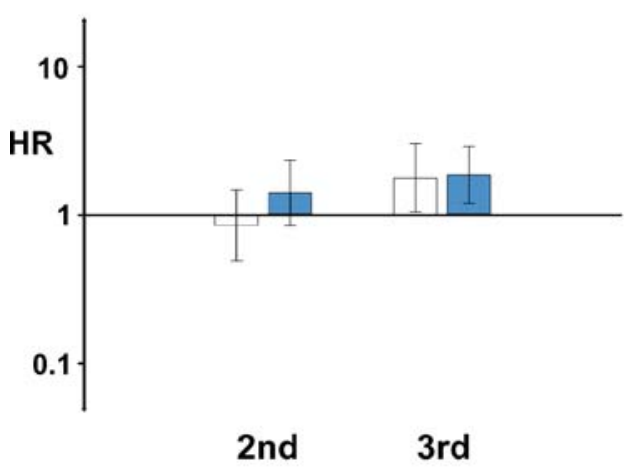

Minimal adjustment b

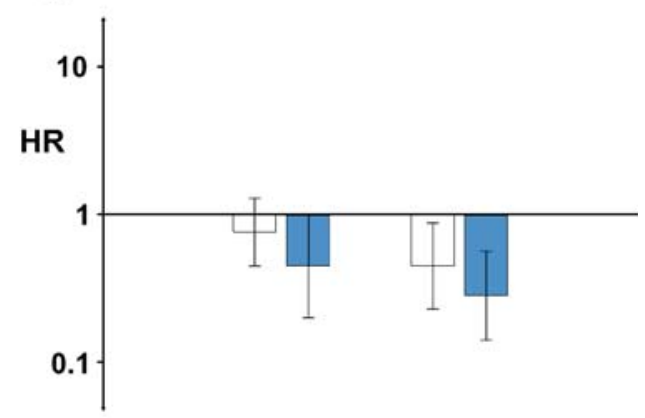

d

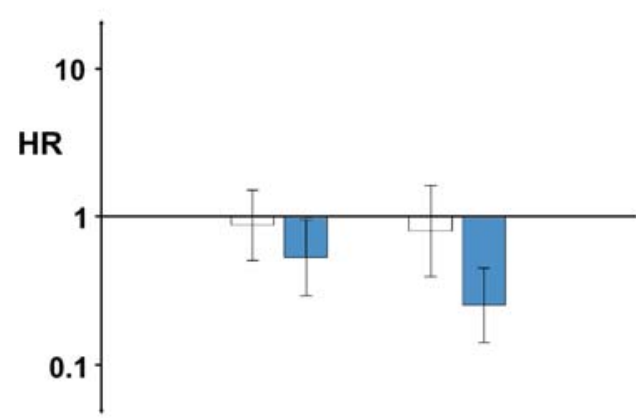

f

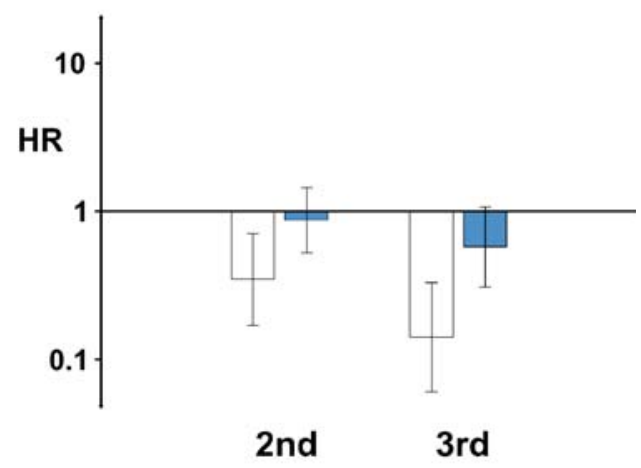

Full adjustment

\section{Leptin tertiles}

difference in mean leptin levels. Second, we adjusted more fully for factors purportedly related to leptin resistance, including central fat deposition [19], hyperinsulinaemia/ insulin resistance $[7,20,26]$ and inflammation markers [21, 27]. Third, as most of our cases were ascertained by fasting glucose, it is possible that their pathogenesis is somewhat different from cases in the other three studies, mostly ascertained by an OGTT. In this regard, dysregulation of hepatic gluconeogenesis in leptin-resistant states may be of particular importance [28, 29].

Leptin levels were markedly increased in obese subjects, and the increased risk of diabetes in those with high leptin levels seen in minimally adjusted models was neutralised after adjusting for obesity indices. Thus, confounding by obesity - a measure of body fat, the source of leptin - appears to explain most of the crude hyperleptinaemia-diabetes association. As additional adjustments unveiled a large protective association, this suggests that the variables included in the models were also confounders of the initial increased risk association. It is possible that such adjustments controlled more fully for factors related to obesity and leptin resistance. The inclusion of baseline glucose, while presented here also as confounding, may well represent over-adjustment, as 
adjusted leptin action at baseline would be expected to lower concurrently measured glucose, and this glucose lowering could mediate part of the protective leptin effect seen in the final model.

Heterogeneity of leptin associations merits discussion. While the pattern of associations was consistent across subgroup categories of sex, ethnicity, smoking and obesity, the decrease in diabetes incidence was minimal in those with impaired fasting glucose (interaction $p=0.04$ ) and was present only in those with higher inflammation scores (interaction $p=0.03$ ). We find no clear explanation in the literature for this heterogeneity. Nonetheless, these findings suggest, first, that leptin's protective actions may be less effective or less important once impaired fasting glucose is established; and second, that leptin, perhaps because one of its functions is to facilitate the inflammatory response [4], may best exert its metabolic effects in a proinflammatory milieu. Although adjusted higher leptin levels predicted less diabetes in both men and women, the association appeared to be stronger, though not statistically significantly so, among women, perhaps reflecting the important role of leptin in female physiology [30]. A physiological basis for our epidemiological findings exists, as leptin, at a molecular level, elicits several actions potentially protective against diabetes. It decreases energy intake, increases energy expenditure, and partitions caloric surplus. With variable effects on glucose homeostasis, overall, leptin appears to improve glucose utilisation [6]. Like adiponectin, leptin activates AMP-activated protein kinase (AMPK), which in turn stimulates glucose uptake through pathways that are independent of insulin signalling $[16,31]$. By promoting fatty acid oxidation, leptin reduces ectopic fat accumulation in non-adipose tissues, thereby increasing insulin sensitivity and decreasing insulin secretion $[13,14,32,33]$. Leptin stimulates expression of the gene for peroxisome proliferator-activator receptor- $\gamma$ co-activator- $1 \alpha$ (PGC- $1 \alpha)$, and upregulation of uncoupling proteins 1 and 2 and mitochondrial biogenesis might offer additional mechanisms for its role in the regulation of caloric surplus [34].

In the liver, leptin increases fatty acid oxidation, probably by repressing stearoyl-CoA desaturase-1 (SCD-1) $[35,36]$. In vivo evidence suggests a role for leptin in the acute regulation of hepatic triglyceride metabolism and triglyceride homeostasis [20]. Resistance to leptin's hepatic actions leads not only to hepatic steatosis [37], but also to increased hepatic gluconeogenesis [28].

Leptin effects on beta cell function are less clear [12]. although leptin has been shown to decrease insulin secretion $[32,33]$, this effect was seen in parallel with others that led to a marked improvement in metabolic parameters in one animal model of diabetes [17]. Its ability to partition caloric surplus to avoid deposition of fat in non-adipose tissues could minimise beta cell lipotoxicity and apoptosis [38].
On balance, we believe that these basic science findings, coupled with our epidemiological data, make it unlikely that leptin itself directly causes diabetes. The more likely alternative is that being overweight, or factors intimately related to the overweight state, such as chronic overeating (and or specific patterns of eating) [39-41] and a sedentary lifestyle [42, 43], lead to leptin resistance with resulting hyperleptinaemia.

Leptin resistance/hyperleptinaemia may result from defects in the transport of leptin through the blood-brain barrier or from increased expression of genes encoding inhibitors of leptin signalling pathways, such as suppressor of cytokine signalling 3 (SOCS3) and protein tyrosine phosphatase 1B (PTP1B) [18]. Additionally, leptin action/ resistance may vary according to different fractions of free and bound leptin and different levels of plasma soluble leptin receptors [41, 44].

Our findings, coupled with the literature, support the hypothesis that leptin resistance, with resultant dysregulation of leptin action, not leptin levels per se, is related to processes, such as altered hepatic metabolism, ectopic fat deposition, lipotoxicity, insulin resistance, impaired insulin secretion and decreased whole-body glucose utilisation, that produce diabetes and other related diseases in humans [45]. Additionally, our data showing higher adjusted levels of leptin in the obese and those with higher inflammation scores (Table 1) support bench research demonstrating that adipocytes and proinflammatory mediators have important roles in stimulating hyperleptinaemia and/or leptin resistance. In fact, the leptin resistance so produced may be an important pathway by which obesity causes diabetes. Additionally, the much higher adjusted levels found in women, as previously reported, attests its special importance in female physiology [30].

Potential limitations to our study merit comment. First, selection bias, either due to participants not returning for follow-up or not having a sample available for measurement, could conceivably have influenced our results. However, we have little a priori reason to believe that the association between leptin and incident diabetes should be stronger or weaker among those without an available sample or who were lost to follow-up. Furthermore, exclusion of some participants with cardiovascular disease has not prevented the demonstration of associations of incident diabetes with factors such as inflammation markers and adiponectin in this sample. Second, epidemiological studies such as ours are restricted in their ability to assess independent effects of inter-related variables, such as leptin, obesity, inflammation, glucose and insulin resistance. Finally, as we ascertained diabetes without use of an OGTT, some misclassification in diabetes ascertainment was likely to have occurred. However, because our follow-up was relatively long, and as, if anything, leptin's protective association was stronger among those with normal fasting glucose than those in whom it was 
impaired, we believe this misclassification is unlikely to have produced associations as a result of reverse causality.

In conclusion, high leptin levels, probably reflecting leptin resistance, predict an increased risk of type 2 diabetes. Yet, once the effects of overall and central obesity, hyperinsulinaemia, inflammation and other metabolic syndrome components are taken into account, high leptin levels predict a decreased risk of diabetes, independently of adiponectin. If this result is confirmed, it validates, at a human population level, bench research suggesting that leptin's basic physiological functions protect against diabetes.

Acknowledgements The authors thank the staff and participants in the ARIC study for their important contributions. The National Institute of Diabetes, Digestive and Kidney Diseases Grant R01-DK056918; National Heart, Lung, and Blood Institute Contracts N01-HC -55015, N01-HC-55016, N01-HC-55018, N01-HC-55019, N01-HC-55020, N01-HC-55021 and N01-HC-55022 provided support for this study. B. B. Duncan and M. I. Schmidt received support from a Centers of Excellence Grant from the CNPq (National Council for Scientific and Technological Development).

Duality of interest The authors state that there is no duality of interest.

\section{References}

1. Schmidt MI, Duncan BB, Sharrett AR et al (1999) Markers of inflammation and prediction of diabetes mellitus in adults (Atherosclerosis Risk in Communities study): a cohort study. Lancet 353:1649-1652

2. Duncan BB, Schmidt MI, Pankow JS et al (2003) Low-grade systemic inflammation and the development of type 2 diabetes: the Atherosclerosis Risk in Communities study. Diabetes 52:1799-1805

3. Duncan BB, Schmidt MI (2006) The epidemiology of low-grade chronic systemic inflammation and type 2 diabetes. Diabetes Technol Ther 8:7-17

4. La Cava A, Matarese G (2004) The weight of leptin in immunity. Nat Rev Immunol 4:371-379

5. Peelman F, Waelput W, Iserentant $\mathrm{H}$ et al (2004) Leptin: linking adipocyte metabolism with cardiovascular and autoimmune diseases. Prog Lipid Res 43:283-301

6. Yildiz BO, Haznedaroglu IC (2006) Rethinking leptin and insulin action: therapeutic opportunities for diabetes. Int J Biochem Cell Biol 38:820-830

7. Ceddia RB (2005) Direct metabolic regulation in skeletal muscle and fat tissue by leptin: implications for glucose and fatty acids homeostasis. Int J Obes (Lond) 29:1175-1183

8. McNeely MJ, Boyko EJ, Weigle DS et al (1999) Association between baseline plasma leptin levels and subsequent development of diabetes in Japanese Americans. Diabetes Care 22:65-70

9. Boyko EJ, de Courten M, Zimmet PZ, Chitson P, Tuomilehto J, Alberti KG (2000) Features of the metabolic syndrome predict higher risk of diabetes and impaired glucose tolerance: a prospective study in Mauritius. Diabetes Care 23:1242-1248

10. Franks PW, Brage S, Luan J et al (2005) Leptin predicts a worsening of the features of the metabolic syndrome independently of obesity. Obes Res 13:1476-1484
11. Ceddia RB, Koistinen HA, Zierath JR, Sweeney G (2002) Analysis of paradoxical observations on the association between leptin and insulin resistance. FASEB J 16:1163-1176

12. Zhao YF, Feng DD, Chen C (2006) Contribution of adipocytederived factors to beta-cell dysfunction in diabetes. Int $\mathrm{J}$ Biochem Cell Biol 38:804-819

13. Shimabukuro M, Koyama K, Chen G et al (1997) Direct antidiabetic effect of leptin through triglyceride depletion of tissues. Proc Natl Acad Sci USA 94:4637-4641

14. Muoio DM, Dohm GL, Fiedorek FT Jr, Tapscott EB, Coleman RA, Dohn GL (1997) Leptin directly alters lipid partitioning in skeletal muscle. Diabetes 46:1360-1363

15. Shimabukuro M, Wang MY, Zhou YT, Newgard CB, Unger RH (1998) Protection against lipoapoptosis of beta cells through leptin-dependent maintenance of Bcl-2 expression. Proc Natl Acad Sci USA 95:9558-9561

16. Minokoshi Y, Kim YB, Peroni OD et al (2002) Leptin stimulates fatty-acid oxidation by activating AMP-activated protein kinase. Nature 415:339-343

17. Toyoshima Y, Gavrilova O, Yakar S et al (2005) Leptin improves insulin resistance and hyperglycemia in a mouse model of type 2 diabetes. Endocrinology 146:4024-4035

18. Munzberg H, Myers MG Jr (2005) Molecular and anatomical determinants of central leptin resistance. Nat Neurosci 8:566-570

19. Tong J, Fujimoto WY, Kahn SE et al (2005) Insulin, C-peptide, and leptin concentrations predict increased visceral adiposity at 5- and 10-year follow-ups in nondiabetic Japanese Americans. Diabetes 54:985-990

20. Huang W, Dedousis N, Bandi A, Lopaschuk GD, O’Doherty RM (2006) Liver triglyceride secretion and lipid oxidative metabolism are rapidly altered by leptin in vivo. Endocrinology 147:1480-1487

21. Yang XP, Schaper F, Teubner A et al (2005) Interleukin-6 plays a crucial role in the hepatic expression of SOCS3 during acute inflammatory processes in vivo. J Hepatol 43:704-710

22. ARIC Investigators (1989) The Atherosclerosis Risk in Communities (ARIC) study. Am J Epidemiol 129:687-702

23. Lin DY (2000) On fitting Cox's proportional hazards models to survey data. Biometrika 87:37-47

24. Collett D (1994) Modelling survival data in medical research. Chapman \& Hall, London

25. Duncan BB, Schmidt MI, Pankow J et al (2004) Adiponectin and the development of type 2 diabetes: the ARIC study. Diabetes 53:2473-2478

26. Benomar Y, Wetzler S, Larue-Achagiotis C, Djiane J, Tome D, Taouis M (2005) In vivo leptin infusion impairs insulin and leptin signalling in liver and hypothalamus. Mol Cell Endocrinol 242:59-66

27. Kirchgessner TG, Uysal KT, Wiesbrock SM, Marino MW, Hotamisligil GS (1997) Tumor necrosis factor-alpha contributes to obesity-related hyperleptinemia by regulating leptin release from adipocytes. J Clin Invest 100:2777-2782

28. Rossetti L, Massillon D, Barzilai N et al (1997) Short term effects of leptin on hepatic gluconeogenesis and in vivo insulin action. J Biol Chem 272:27758-27763

29. Brabant G, Muller G, Horn R, Anderwald C, Roden M, Nave H (2005) Hepatic leptin signaling in obesity. FASEB J 19:1048-1050

30. Sagawa N, Yura S, Itoh H et al (2002) Possible role of placental leptin in pregnancy: a review. Endocrine 19:65-71

31. Guerre-Millo M (2003) Extending the glucose/fatty acid cycle: a glucose/adipose tissue cycle. Biochem Soc Trans 31:1161-1164

32. Emilsson V, Liu YL, Cawthorne MA, Morton NM, Davenport M (1997) Expression of the functional leptin receptor mRNA in pancreatic islets and direct inhibitory action of leptin on insulin secretion. Diabetes 46:313-316

33. Kieffer TJ, Heller RS, Leech CA, Holz GG, Habener JF (1997) Leptin suppression of insulin secretion by the activation of ATP-sensitive $\mathrm{K}+$ channels in pancreatic beta-cells. Diabetes 46:1087-1093 
34. Orci L, Cook WS, Ravazzola M et al (2004) Rapid transformation of white adipocytes into fat-oxidizing machines. Proc Natl Acad Sci USA 101:2058-2063

35. Cohen P, Friedman JM (2004) Leptin and the control of metabolism: role for stearoyl-CoA desaturase-1 (SCD-1). J Nutr 134:2455S-2463S

36. Cohen P, Ntambi JM, Friedman JM (2003) Stearoyl-CoA desaturase-1 and the metabolic syndrome. Curr Drug Targets Immune Endocr Metabol Disord 3:271-280

37. Barzilai N, Wang J, Massilon D, Vuguin P, Hawkins M, Rossetti L (1997) Leptin selectively decreases visceral adiposity and enhances insulin action. J Clin Invest 100:3105-3110

38. Unger RH (2003) Minireview: weapons of lean body mass destruction: the role of ectopic lipids in the metabolic syndrome. Endocrinology 144:5159-5165

39. Park SY, Cho YR, Kim HJ et al (2005) Unraveling the temporal pattern of diet-induced insulin resistance in individual organs and cardiac dysfunction in C57BL/6 mice. Diabetes 54:3530-3540

40. Jonsson T, Olsson S, Ahren B, Bog-Hansen TC, Dole A, Lindeberg S (2005) Agrarian diet and diseases of affluence-do evolutionary novel dietary lectins cause leptin resistance? BMC Endocr Disord 5:10

41. Magni P, Liuzzi A, Ruscica M et al (2005) Free and bound plasma leptin in normal weight and obese men and women: relationship with body composition, resting energy expenditure, insulinsensitivity, lipid profile and macronutrient preference. Clin Endocrinol (Oxf) 62:189-196

42. Fox C, Esparza J, Nicolson M et al (1999) Plasma leptin concentrations in Pima Indians living in drastically different environments. Diabetes Care 22:413-417

43. Franks PW, Farooqi IS, Luan J et al (2003) Does physical activity energy expenditure explain the between-individual variation in plasma leptin concentrations after adjusting for differences in body composition? J Clin Endocrinol Metab 88:3258-3263

44. Cohen P, Yang G, Yu X et al (2005) Induction of leptin receptor expression in the liver by leptin and food deprivation. J Biol Chem 280:10034-10039

45. Unger RH (2005) Longevity, lipotoxicity and leptin: the adipocyte defense against feasting and famine. Biochimie 87:57-64 